\title{
APPROXIMATION OF SINGULARLY PERTURBED PARABOLIC REACTION-DIFFUSION EQUATIONS WITH NONSMOOTH DATA ${ }^{1}$
}

\author{
GRIGORII I. SHISHKIN \\ Institute of Mathematics and Mechanics, Ural Branch of Russian Academy of Sciences \\ 16 S. Kovalevskaya Str., 620219 Ekaterinburg, Russia \\ E-mail: Grigorii@shishkin.ural.ru
}

\begin{abstract}
In this paper we consider the Dirichlet problem on a rectangle for singularly perturbed parabolic equations of reaction-diffusion type. The reduced (for $\varepsilon=0$ ) equation is an ordinary differential equation with respect to the time variable; the singular perturbation parameter $\varepsilon$ may take arbitrary values from the half-interval $(0,1]$. Assume that sufficiently weak conditions are imposed upon the coefficients and the right-hand side of the equation, and also the boundary function. More precisely, the data satisfy the Hölder continuity condition with a small exponent $\alpha$ and $\alpha / 2$ with respect to the space and time variables. To solve the problem, we use the known $\varepsilon$-uniform numerical method (i.e., a standard finite difference operator on piecewiseuniform fitted meshes over the axes $x_{1}$ and $x_{2}$ ) which was developed previously for problems with sufficiently smooth and compatible data. It is shown that the numerical solution converges $\varepsilon$-uniformly at the rate of $\mathcal{O}\left(N^{-\nu}+N_{0}^{-\nu}\right), \nu=m \alpha^{2}$; here the values of $N$ and $N_{0}$ define the number of nodes in the space (with respect to each variable) and time meshes. We discuss also the behavior of local accuracy of the scheme in the case where the data of the boundary-value problem are smoother on a part of the domain of definition.
\end{abstract}

2000 Mathematics Subject Classification: 65N06; 65N22; 35B25.

Keywords: singular perturbation, finite difference scheme, fitted mesh, $\varepsilon$-uniform convergence.

\section{Introduction}

As is known, depending on the data of a boundary-value problem, in particular, on their smoothness, the solutions of singularly perturbed problems can be sufficiently smooth for each fixed value of the perturbation parameter $\varepsilon$ multiplying the highest derivatives. However, the derivatives of solutions grow without bounds (in boundary and transient layers) as the parameter $\varepsilon$ tends to zero. This is the reason why numerical methods developed for regular problems (see, e.g. [7,9]) yield errors which depend on an inverse power of the perturbation parameter $\varepsilon$; such errors become large for small $\varepsilon$ which is clearly unsatisfactory.

\footnotetext{
${ }^{1}$ The work was supported by the Russian Foundation for Basic Research under grant No. 98-01-00362 and partially by the Enterprise Ireland grant SC-98-612.
} 
For certain problems having sufficiently smooth solutions, $\varepsilon$-uniform numerical methods which converge irrespective of $\varepsilon$ have been developed and thoroughly studied (see, e.g., $[1,10]$ and the references therein) with the convergence analysis given in the maximum norm. However, conditions imposed on the problem data (precisely, on their smoothness and the order of compatibility conditions on nonsmooth parts of the boundary), which ensures that the solution is sufficiently smooth, are restrictive and, as a rule, are substantially overstated so as to make it difficult to use these methods in practice. For example, in [13] (see also $[3,8,10])$ for a reaction-diffusion problem a sufficiently high level of smoothness of the coefficients and source terms (from the class $C^{l}(\bar{G}), l>6$ ) and the boundary functions (from the class $C^{l}\left(\bar{S}_{j}\right) \cap C(S), l>6$, where $S_{j}$ are the sides forming the boundary $S$ of the set $G$ ) was required in order to comply with sufficient conditions of $\varepsilon$-uniform convergence. If the fulfilment of the compatibility conditions at the edges (in three dimensions) or corners (in two dimensions) from $S$ is not assumed, except continuity of the boundary function, then the order of $\varepsilon$-uniform convergence for the scheme on piecewise uniform meshes which was studied in [13] is $\mathcal{O}\left(\left(N^{-1} \ln N\right)^{\nu}+N_{0}^{-\nu}\right), \nu=5^{-1}$. However, if we impose sufficiently restrictive compatibility conditions, which imply the required smoothness of the solution $u(x, t)$ and its regular and singular components from the asymptotic expansion, then the scheme converges with the rate $\mathcal{O}\left(N^{-1} \ln N+N_{0}^{-1}\right)$. In [12] the difference scheme on graded meshes was examined, where the sufficiently high level of smoothness of the problem data (with sufficient regularity and compatibility conditions) was also required to prove $\varepsilon$-uniform convergence. It should be noted that fairly well-known difficulties arise in defining the data which satisfy the required compatibility conditions for singularly perturbed problems (see, e.g., [10] in the case of convection-diffusion equations).

For a recent account of this theory for singularly perturbed elliptic convection-diffusion equations on a rectangle $\bar{D}$, the reader can be referred to [14], in which a special scheme is examined for $u \in C^{2+\alpha}(D) \cup C^{\alpha}(\bar{D})$, where $\alpha$ is any number from $(0,1)$. Some related numerical results were discussed for a corner layer in [3; Chapter 5]. It should be noted that the nature of reaction-diffusion and convection-diffusion problems is quite different. For example, even in the case of constant coefficient equations in one dimension, if the righthand side function $f(x), x \in \bar{d} \equiv[0,1]$ satisfies the condition $f \in C^{\alpha}(\bar{d}), \alpha>0$, then the derivative $u^{(2+\alpha)}(x)$ is bounded on $\bar{d}$ for regular equations of both convection-diffusion and reaction-diffusion types. But in the case of singularly perturbed equations we have $\varepsilon$-uniform boundedness of the derivative $u^{(1+\alpha)}(x), x \in \bar{d}$ for convection-diffusion equations (where the layer appears in a neighborhood of the outflow boundary) and of only the derivative $u^{(\alpha)}(x)$, $x \in \bar{d}$ for reaction-diffusion equations (the layer occurs in a neighborhood of both endpoints of the segment $\bar{d}$ ). Thus, the technique of [14] and the results of this earlier work cannot be simply applied to a reaction-diffusion problem for the singular perturbation case.

Singularly perturbed problems with a low level of smoothness of the data (this implies a lowered smoothness of the derivatives of the solution) can appear, for example, in modeling heat and mass transfer in media with not quite regular physical characteristics. We emphasize that almost no attempt has been made earlier to develop $\varepsilon$-uniform numerical methods for various problems with nonsmooth data, provided that classical solutions still exist. By the above argument, the following theoretical problem appears: find sufficiently weak conditions to be imposed on the involved data, under which classical solutions (for fixed values of the parameter $\varepsilon$ ) do exist, and to examine rigorously $\varepsilon$-uniform numerical methods for a wide class of singularly perturbed reaction-diffusion problems with the aforesaid properties. Thus, it would be of theoretical and practical interest to weaken the requirements imposed 
on the data of relevant problems.

In this paper we consider the Dirichlet problem for singularly perturbed parabolic reaction-diffusion equations in the case of a rectangular domain. The reduced equations (for $\varepsilon=0$ ) are ordinary differential ones of first order with respect to the time variable. The coefficients and the right-hand side, and also the boundary function satisfy Hölder conditions of a small order $\alpha \in(0,1)$. This assumption provides that the solution belongs to the class $C^{2+\alpha, 1+\alpha / 2}(G) \cap C^{\alpha, \alpha / 2}(\bar{G}), G=D \times(0, T]$. As the parameter $\varepsilon$ tends to zero, such problems exhibit a parabolic boundary layer which is one-dimensional in a neighborhood of smooth parts of the boundary and two-dimensional at corner points of the rectangle. We place more emphasis on the asymptotic nature of the boundary layers in order to obtain a-priori estimates in the right form for proving $\varepsilon$-uniform convergence of a numerical method, with minimal restrictions on the smoothness of the problem data and no compatibility conditions imposed.

Under the above-mentioned conditions, the known finite difference scheme on piecewise uniform meshes (see, e.g., $[1,8,13]$ for the description of fitted mesh methods), which was constructed in [13] for problems with sufficient smoothness of the data and sufficiently strong compatibility conditions, is shown to converge $\varepsilon$-uniformly with the rate $\mathcal{O}\left(N^{-\nu}+N_{0}^{-\nu}\right)$, in the maximum norm, where $\nu=m \alpha^{2}$. Note that the use of the maximum norm is essential because other weaker norms, for example the energy norm, are inappropriate for detecting the boundary and interior layers in the solutions of singularly perturbed reaction-diffusion problems (see, e.g., [3; Section 1.2]). To emphasize how strongly the smoothness of the data influences the accuracy of the scheme, we consider the case when the data of the original problem on some subdomain $\bar{G}^{0}$ are smoother in comparison with the same data on the whole domain $\bar{G}$. As is shown, this leads to the improvement of local accuracy of the numerical solution (on the set which is strictly interior to this subdomain $\bar{G}^{0}$ ) that is very significant in applications. It would be of practically certain interest to verify the theoretical results with numerical experiments, as an important adjunct to the systematic study of special $\varepsilon$-uniform schemes for reaction-diffusion problems with nonsmooth data, but this topic is beyond the scope of the present paper.

\section{Problem formulation}

On the rectangle $\bar{D}$, where $D=\left\{x: 0<x_{s}<d_{s}, s=1,2\right\}$, we consider the boundary-value problem for the parabolic equation ${ }^{2}$

$$
\begin{aligned}
L_{(1.1)} u(x, t) & =f(x, t), & & (x, t) \in G, \\
u(x, t) & =\varphi(x, t), & & (x, t) \in S .
\end{aligned}
$$

Here

$$
\begin{gathered}
G=D \times(0, T], \quad S=\bar{G} \backslash G, \\
L_{(1.1)} \equiv \varepsilon^{2} \sum_{s=1,2} a_{s}(x, t) \frac{\partial^{2}}{\partial x_{s}^{2}}-c(x, t)-p(x, t) \frac{\partial}{\partial t},
\end{gathered}
$$

the functions $a_{s}(x, t), c(x, t), p(x, t), f(x, t), s=1,2$, and also the boundary function $\varphi(x, t)$ possess some smoothness on the set $\bar{G}$ and on the sides of the set $G$ (from $S$ ), respectively,

\footnotetext{
${ }^{2}$ The notation $L_{(j . k)}, M_{(j . k)}$ or $f_{(j . k)}(x, t)$ means that this operator, constant or function is introduced in the formula $(j . k)$.
} 
the function $\varphi(x, t)$ is continuous on $S$. The coefficients of the differential equation are assumed to satisfy the condition

$$
a_{s}(x, t) \geqslant a_{0}, \quad p(x, t) \geqslant p_{0}, \quad c(x, t) \geqslant 0, \quad(x, t) \in \bar{G}, \quad s=1,2, \quad a_{0}, p_{0}>0 .
$$

The parameter $\varepsilon$ takes arbitrary values from the half-interval $(0,1]$. The solution of this problem is regarded in the classical sense, as a function $u \in C^{2,1}(G) \cap C(\bar{G})$ satisfying Eq. (1.1a) on $G$ and the boundary condition (1.1b) on $S$.

The data of problem (1.1) are assumed to be smooth enough so that the solution on $\bar{G}$ and its derivatives on $G$ involved in (1.1a) satisfy the Hölder condition of a sufficiently small order $\alpha(0<\alpha<1)$ for each fixed value of the parameter $\varepsilon$, i.e., (see [6])

$$
\begin{gathered}
\sup _{(x, t),\left(x^{\prime}, t\right) \in \bar{G}}\left\{\left|x-x^{\prime}\right|^{-\alpha}\left|u(x, t)-u\left(x^{\prime}, t\right)\right|\right\}, \\
\sup _{(x, t),\left(x, t^{\prime}\right) \in \bar{G}}\left\{\left|t-t^{\prime}\right|^{-\alpha / 2}\left|u(x, t)-u\left(x, t^{\prime}\right)\right|\right\} \leqslant C(\varepsilon) ; \\
\sup _{\substack{(x, t),\left(x^{\prime}, t\right) \in G \\
\rho\left((x, t),\left(x^{\prime}, t^{\prime}\right)\right) \geqslant r}}\left\{\left|x-x^{\prime}\right|^{-\alpha_{0}}\left|u(x, t)-u\left(x^{\prime}, t\right)\right|\right\}, \\
\sup _{\substack{(x, t),\left(x, t^{\prime}\right) \in G \\
\rho\left((x, t),\left(x^{\prime}, t^{\prime}\right)\right) \geqslant r}}\left\{\left.\left|t-t^{\prime}\right|\right|^{-\alpha_{0} / 2}\left|u(x, t)-u\left(x, t^{\prime}\right)\right|\right\} \leqslant C_{1}(r, \varepsilon) ; \\
\sup _{\substack{(x, t),\left(x^{\prime}, t\right) \in G \\
\rho\left((x, t),\left(x^{\prime}, t^{\prime}\right)\right) \geqslant r}}\left\{\left|x-x^{\prime}\right|^{-\alpha}\left|\frac{\partial^{k+k_{0}}}{\partial x_{1}^{k_{1}} \partial x_{2}^{k_{2}} \partial t^{k_{0}}}\left(u(x, t)-u\left(x^{\prime}, t\right)\right)\right|\right\}, \\
\sup _{(x, t),\left(x, t^{\prime}\right) \in G}\left\{\left|t-t^{\prime}\right|^{-\alpha / 2}\left|\frac{\partial^{k+k_{0}}}{\partial x_{1}^{k_{1}} \partial x_{2}^{k_{2}} \partial t^{k_{0}}}\left(u(x, t)-u\left(x, t^{\prime}\right)\right)\right|\right\} \leqslant C_{2}(r, \varepsilon), \\
\rho\left((x, t),\left(x^{\prime}, t^{\prime}\right)\right) \geqslant r
\end{gathered}
$$

where $k=k_{1}+k_{2}, k+2 k_{0}=2, \alpha_{0}>\alpha$, and $\rho\left((x, t),\left(x^{\prime}, t^{\prime}\right)\right)$ is a distance between the boundary $S$ and either the set of the points $\left\{(x, t),\left(x^{\prime}, t\right)\right\}$ or the set of the points $\left\{(x, t),\left(x, t^{\prime}\right)\right\}$, for example,

$$
\rho\left((x, t),\left(x^{\prime}, t^{\prime}\right)\right)=\min _{\left(x^{*}, t^{*}\right) \in S}\left\{\left|x-x^{*}\right|+\left|t-t^{*}\right|^{1 / 2},\left|x^{\prime}-x^{*}\right|+\left|t^{\prime}-t^{*}\right|^{1 / 2}\right\},
$$

the constant $C_{i}(r, \varepsilon)$ increases when $r$ tends to zero; $r>0$ is an arbitrary number. The fulfilment of compatibility conditions at the edges from $S_{E}$, except the continuity of the boundary function, is not assumed. Here and below $S=S_{0} \cup S^{L}$, where $S_{0}=\bar{D} \times\{t=0\}$ and $S^{L}=\Gamma \times(0, T]$ denote the lower base and the lateral faces of the set $G, \Gamma=\bar{D} \backslash D$; $S_{E}=\{\Gamma \times\{t=0\}\} \cup\left\{\Gamma_{E} \times(0, T]\right\}$ consists of the lower and lateral edges of $G, \Gamma_{E}$ is the set of corner points of $D$.

As the parameter $\varepsilon$ tends to zero, a parabolic boundary layer appears in a neighborhood of the set $S^{L}$.

Assume that the data of problem (1.1) satisfy the conditions

$$
a_{s}, c, p, f \in C^{\alpha, \alpha / 2}(\bar{G}), \quad \varphi \in C^{\alpha, \alpha / 2}\left(\bar{S}_{j}\right) \cap C(S), \quad \alpha \in(0,1), \quad s=1,2, \quad j=0,1, \ldots, 4,
$$

where $S_{j}=\Gamma_{j} \times(0, T], j=1,2,3,4$ are the lateral faces of $G$; the sides $\Gamma_{s}, \Gamma_{s+2}$ of the rectangle $D$ are orthogonal to the $x_{s}$ axis, $s=1,2 ; \Gamma_{1}$ and $\Gamma_{2}$ pass through the point $(0,0)$; let $\Gamma_{j}=\bar{\Gamma}_{j}$. 
Condition (1.2) ensures the inclusion $u \in C^{2+\alpha,(2+\alpha) / 2}(G) \cap C^{\alpha, \alpha / 2}(\bar{G})$ for each fixed value of the parameter $\varepsilon$; but if (1.2) is violated, the solution $u$ of problem (1.1), generally speaking, is not classical, $u \notin C^{2,1}(G) \cap C(\bar{G})$ (see, e.g., [6]).

To solve the boundary-value problem (1.1) under the above conditions, as has been already noted in the Introduction, we are interested in numerical methods which produce $\varepsilon$-uniform approximations to $u$. One of these $\varepsilon$-uniform methods was constructed in [13] for problems with sufficiently smooth and compatible data; namely, the difference scheme comprising a monotone classical approximation [7,11] of problem $(1.1)$ on piecewise-uniform meshes fitted to the boundary layers. It turns out that this scheme converges $\varepsilon$-uniformly. This assertion is proved in Section 3. Appropriate decompositions of the solution $u$ into regular and singular parts and a-priori estimates for these components in the right form, necessary for the convergence proof, are given in Section 2.

\section{A-priori estimates}

When deriving a-priori estimates for the solutions of problem (1.1), (1.2), we approximate this problem by auxiliary boundary-value problems with the smoothed data. For the solutions of these auxiliary problems, which are similar to problem (1.1), (1.2), we give their specific representations, and also we obtain a number of a-priori estimates for the components from these representations. The estimation technique is a development of that from [13] (see also $[3,8,14]$; in [8] and [3] the detailed technique of deriving a-priori estimates and the estimates themselves are considered solely in the case of sufficiently smooth data). The auxiliary problems with smoother data are being constructed using the Steklov-type averaged data of problem (1.1), (1.2) (the technique of the Steklov smoothing can be found, for example, in [15] and [16; Part I, Chapter I]).

We shall assume that the following condition is fulfulled:

$$
a_{s}, c, p, f \in C^{\alpha_{1}}(\bar{G}), \quad \varphi \in C^{\alpha_{2}}\left(\bar{S}_{j}\right) \cap C(S), \quad s=1,2, \quad j=0,1, \ldots, 4,
$$

where $\alpha_{1}, \alpha_{2}$ are arbitrary numbers satisfying $\alpha_{1}, \alpha_{2} \in(0,1)$. The data of problem (1.1) in the case of condition (2.1) (condition (1.2)) satisfy condition (1.2) (condition (2.1)) for $\alpha \leqslant \min \left[\alpha_{1}, \alpha_{2}\right]$ (respectively, for $\alpha_{1}, \alpha_{2} \leqslant 2^{-1} \alpha$ ), where $\alpha=\alpha_{(1.2)}$.

\subsection{Solution decomposition}

In this subsection we find estimates when the values of the parameter $\varepsilon$ are not too small. We shall consider the solution of some auxiliary problem similar to problem (1.1), which involves a smooth boundary function approximating the original function $\varphi(x, t)$.

Let $\varphi^{1}(x, t)=\varphi^{1}\left(x, t ; \sigma_{0}\right),(x, t) \in S$ be a smooth approximation to the function $\varphi(x, t)$. Here $\varphi^{1}(x, t)$ is the Steklov averaging of the function $v_{\varphi}(x, t),(x, t) \in R^{3}$ with kernel on a support of diameter $\sigma_{0}$; moreover, $v_{\varphi}(x, t)=\varphi(x, t),(x, t) \in S, v_{\varphi} \in C^{\alpha_{2}}\left(\bar{G}^{m}\right)$, where $\bar{G}^{m}$ is an $m$-neighborhood of the set $\bar{G}$; the value of $\sigma_{0}$ is chosen below in Section 3 (see (3.5)). Then the following bounds are valid ${ }^{3}$ :

$$
\begin{aligned}
& \left|\varphi(x, t)-\varphi^{1}(x, t)\right| \leqslant M \sigma_{0}^{\alpha_{2}}, \quad(x, t) \in S, \\
& \left|\frac{\partial^{k+k_{0}}}{\partial x_{1}^{k_{1}} \partial x_{2}^{k_{2}} \partial t^{k_{0}}} \varphi^{1}(x, t)\right| \leqslant M \sigma_{0}^{-k-k_{0}}, \quad(x, t) \in \bar{S}_{j}, \\
& k_{1}+k_{2}=k, \quad k_{s}=k_{s}(k, j), \quad s=0,1,2, \quad j=0,1, \ldots, 4,
\end{aligned}
$$

\footnotetext{
${ }^{3}$ By $M, M_{i}$ (or $m, m_{i}$ ) we denote sufficiently large (small) positive constants independent of $\varepsilon$ and the discretization parameters.
} 
where $k_{1}=k, k_{0} \geqslant 0$ for $j=2,4 ; k_{2}=k, k_{0} \geqslant 0$ for $j=1,3 ; k_{0}=0, k_{1}, k_{2} \geqslant 0$ for $j=0$.

Let $u^{1}(x, t),(x, t) \in \bar{G}$ be a solution of the boundary-value problem

$$
\begin{aligned}
L_{(1.1)} u^{1}(x, t) & =f(x, t), & & (x, t) \in G, \\
u^{1}(x, t) & =\varphi^{1}(x, t), & & (x, t) \in S .
\end{aligned}
$$

The solution of problem (1.1), (2.1) can be decomposed into two functions

$$
u(x, t)=u^{1}(x, t)+v^{1}(x, t), \quad(x, t) \in \bar{G},
$$

where $u^{1}(x, t)=u^{1}\left(x, t ; \alpha_{1}, \alpha_{2}, \sigma_{0}\right)$, and $v^{1}(x, t)=v^{1}\left(x, t ; \alpha_{1}, \alpha_{2}, \sigma_{0}\right)$ is the "remainder" term. The functions $u^{1}(x, t)$ and $v^{1}(x, t)$ satisfy the estimates

$$
\left|u(x, t)-u^{1}(x, t)\right|, \quad\left|v^{1}(x, t)\right| \leqslant M \sigma_{0}^{\alpha_{2}}, \quad(x, t) \in \bar{G} .
$$

In problem (2.3) we pass to the stretched variables $\xi=\xi(x, \varepsilon), t ; \xi_{s}=\varepsilon^{-1} x_{s}, s=1,2$. Taking account of inner a-priori estimates [6], we find

$\left|\frac{\partial^{k+k_{0}}}{\partial \xi_{1}^{k_{1}} \partial \xi_{2}^{k_{2}} \partial t^{k_{0}}} \widetilde{u}^{1}(\xi, t)\right| \leqslant M\left[\widetilde{r}^{-1}+t^{-1 / 2}\right]^{k+2 k_{0}}, \quad(\xi, t) \in \widetilde{G}, \quad k=k_{1}+k_{2}, \quad k+2 k_{0} \leqslant 2+\alpha_{1}$.

Here $\widetilde{r}=\varepsilon^{-1} r, r=r(x)$ is the distance from the point $x$ to the boundary $\Gamma, \widetilde{G}$ is the image of the set $G, \widetilde{v}(\xi, t)=v(x(\xi), t), \alpha_{i}=\alpha_{i(2.1)}$. Returning to the original variables, we obtain

$$
\left|\frac{\partial^{k+k_{0}}}{\partial x_{1}^{k_{1}} \partial x_{2}^{k_{2}} \partial t^{k_{0}}} u^{1}(x, t)\right| \leqslant M \varepsilon^{-k}\left[\varepsilon r^{-1}+t^{-1 / 2}\right]^{k+2 k_{0}}, \quad(x, t) \in G, \quad k+2 k_{0} \leqslant 2+\alpha_{1} .
$$

By using majorant functions we get the estimate

$$
\left|u^{1}(x, t)-\varphi^{1}\left(x^{*}, t^{*}\right)\right| \leqslant M\left(\varepsilon+\sigma_{0}^{1 / 2}\right) \sigma_{0}^{-1} \min \left[\varepsilon^{-1} r,\left(\varepsilon+\sigma_{0}^{1 / 2}\right) \sigma_{0}^{-1} t\right], \quad(x, t) \in \bar{G},
$$

where $\left(x^{*}, t^{*}\right) \in S,\left(x^{*}, t^{*}\right)=\left(x^{*}(x, t), t^{*}(x, t)\right)$ is a point on $S$ nearest to the point $(x, t)$ (in the sense of the distance $\left.\left(x_{1}^{2}+x_{2}^{2}+t^{2}\right)^{1 / 2}\right)$. If more of such points exist, then $\left(x^{*}, t^{*}\right)$ is one of them.

Thus, we have decomposed the solution of problem (1.1) in the form (2.4), where the function $u^{1}(x, t)$ is "sufficiently" smooth inside $G$ and continuous (satisfying the Lipschitz condition) with respect to the distance to the boundary $S$, and the function $v^{1}(x, t)$ is sufficiently small on $\bar{G}$. The smoothness of the function $u^{1}(x, t)$ and its Lipschitz constant (see the estimate $(2.7)$ ), and also the smallness of the function $v^{1}(x, t)$ are defined by the four parameters $\varepsilon, \sigma_{0}, \alpha_{1}$ and $\alpha_{2}$.

Theorem 2.1. Let the smoothness condition (2.1) hold. Then the estimates (2.5)-(2.7) are valid for the functions $u_{(2.3)}^{1}(x, t)$ and $v_{(2.4)}^{1}(x, t)$ from representation $(2.4)$ of the solution of problem (1.1).

\subsection{Bounds on the regular and singular components of the solution}

In this subsection we obtain a number of estimates based on the asymptotic behavior of the solution. Note that the traditional asymptotic analysis requires that the problem data are sufficiently smooth (smoother than the conditions in (2.1)), and also the terms of the asymptotic expansion (in powers of $\varepsilon$ ) are smooth (see, e.g., [5]). This is too restrictive, because engineers often have problems in the applications that do not satisfy such strong smoothness conditions. As in Section 2.1, we shall consider the solution of some auxiliary boundary-value problem with smooth data which approximate the data of problem (1.1), $(2.1)$. 


\subsubsection{Let}

$$
\begin{array}{lll}
a_{s}^{*}(x, t)=a_{s}^{*}\left(x, t ; \sigma_{1}\right), & c^{*}(x, t)=c^{*}\left(x, t ; \sigma_{1}\right), & \\
p^{*}(x, t)=p^{*}\left(x, t ; \sigma_{1}\right), & f^{*}(x, t)=f^{*}\left(x, t ; \sigma_{1}\right), & (x, t) \in \bar{G} ; \\
\varphi^{*}(x, t)=\varphi^{*}\left(x, t ; \sigma_{2}\right), & & (x, t) \in S ; \quad s=1,2,
\end{array}
$$

be smooth approximations, respectively, to the functions $a_{s}(x, t), c(x, t), p(x, t), f(x, t)$ and $\varphi(x, t)$ (the Steklov averaging of the function $\varphi(x, t),(x, t) \in S$, and also of prolongations of the functions $a_{s}(x, t), c(x, t), p(x, t), f(x, t)$ beyond an $m$-neighborhood of the set $\bar{G}$ with retention of their properties; $\sigma_{1}$ and $\sigma_{2}$ are the diameters of the support for the averaging kernel). The values of $\sigma_{1}$ and $\sigma_{2}$ are chosen below when we will derive estimates (2.18)-(2.20) and (3.13)-(3.15). Then the following relations are valid:

$$
\begin{gathered}
\left|a_{s}(x, t)-a_{s}^{*}(x, t)\right|, \ldots,\left|f(x, t)-f^{*}(x, t)\right| \leqslant M \sigma_{1}^{\alpha_{1}}, \\
\left|\frac{\partial^{k+k_{0}}}{\partial x_{1}^{k_{1}} \partial x_{2}^{k_{2}} \partial t^{k_{0}}} a_{s}^{*}(x, t)\right|, \ldots,\left|\frac{\partial^{k+k_{0}}}{\partial x_{1}^{k_{1}} \partial x_{2}^{k_{2}} \partial t^{k_{0}}} f^{*}(x, t)\right| \leqslant M \sigma_{1}^{-k-k_{0}}, \quad(x, t) \in \bar{G} \\
\mid \frac{\left|\varphi(x, t)-\varphi^{*}(x, t)\right| \leqslant M \sigma_{2}^{\alpha_{2}}, \quad(x, t) \in S}{\left|\frac{\partial^{\bar{k}+\bar{k}_{0}}}{\partial x_{1}^{\bar{k}_{1}} \partial x_{2}^{\bar{k}_{2}} \partial t^{\bar{k}_{0}}} \varphi^{*}(x, t)\right| \leqslant M \sigma_{2}^{-\bar{k}-\bar{k}_{0}}, \quad(x, t) \in \bar{S}_{j},} \\
\bar{k}_{s}=k_{s(2.2)}(\bar{k}, j) .
\end{gathered}
$$

By $u^{*}(x, t),(x, t) \in \bar{G}$ we denote the solution of the boundary-value problem

$$
\begin{aligned}
L_{(2.10)} u^{*}(x, t) & =f^{*}(x, t), \quad(x, t) \in G, \\
u^{*}(x, t) & =\varphi^{*}(x, t), \quad(x, t) \in S,
\end{aligned}
$$

where

$$
L_{(2.10)} \equiv \varepsilon^{2} \sum_{s=1,2} a_{s}^{*}(x, t) \frac{\partial^{2}}{\partial x_{s}^{2}}-c^{*}(x, t)-p^{*}(x, t) \frac{\partial}{\partial t} .
$$

We proceed by observing that the solution of problem (2.10) can be written in the form

$$
\begin{aligned}
u^{*}(x, t) & =U^{*}(x, t)+\sum_{j=1}^{4} V_{j}^{*}(x, t)+\sum_{j=1}^{4} V_{j i}^{*}(x, t)+v^{*}(x, t) \\
& \equiv U^{*}(x, t)+V^{*}(x, t)+v^{*}(x, t), \quad(x, t) \in \bar{G},
\end{aligned}
$$

that is, we decompose $u^{*}(x, t)$ into its "smooth", $U^{*}(x, t)$, and singular, $V^{*}(x, t)$, parts with the remainder term $v^{*}(x, t)$. The functions $V_{j}^{*}(x, t)$ and $V_{j i}^{*}(x, t)$ are respectively onedimensional and corner parabolic layers. The components from (2.11) are the solutions of the problems

$$
\begin{aligned}
L_{(2.12)} U^{*}(x, t) & \equiv\left\{-c^{*}(x, t)-p^{*}(x, t) \frac{\partial}{\partial t}\right\} U^{*}(x, t)=f^{*}(x, t), & & (x, t) \in \bar{G} \backslash S_{0}, \\
U^{*}(x, t) & =\varphi^{*}(x, t), & (x, t) & \in S_{0}
\end{aligned}
$$




$$
\begin{aligned}
& L_{(2.13)} V_{1}^{*}(x, t) \equiv\left\{\varepsilon^{2} a_{1}^{*}\left(0, x_{2}, t\right) \frac{\partial^{2}}{\partial x_{1}^{2}}-c^{*}\left(0, x_{2}, t\right)-p^{*}\left(0, x_{2}, t\right) \frac{\partial}{\partial t}\right\} V_{1}^{*}(x, t)=0,(x, t) \in \bar{G} \backslash\left\{S_{0} \cup \bar{S}_{1} \cup \bar{S}_{3}\right\}, \\
& V_{1}^{*}(x, t)=\varphi^{*}(x, t)-U^{*}(x, t),(x, t) \in \bar{S}_{1}, \\
& V_{1}^{*}(x, t)=0,(x, t) \in S_{0} \cup \bar{S}_{3} ; \\
& L_{(2.14)} V_{12}^{*}(x, t) \equiv \begin{cases}\left.\varepsilon^{2} \sum_{s=1,2} a_{s}^{*}(0,0, t) \frac{\partial^{2}}{\partial x_{s}^{2}}-c^{*}(0,0, t)-p^{*}(0,0, t) \frac{\partial}{\partial t}\right\} V_{12}^{*}(x, t)=0, \\
V_{12}^{*}(x, t)=\varphi^{*}(x, t)-\left(U^{*}(x, t)+V_{1}^{*}(x, t)+V_{2}^{*}(x, t)\right), & (x, t) \in \bar{S}_{1} \cup \bar{S}_{2}, \\
V_{12}^{*}(x, t)=0, & (x, t) \in S_{0} \cup \bar{S}_{3} \cup \bar{S}_{4} ;\end{cases}
\end{aligned}
$$

the functions $V_{j}^{*}(x, t)$ and $V_{j i}^{*}(x, t), j=2,3,4$ are the solutions of problems similar to (2.13) and (2.14).

Remark 1. The function $\varphi_{(2.8)}^{*}(x, t)$ can be "improved" so that the above problems for the functions $U^{*}(x, t), V_{j}^{*}(x, t), V_{j i}^{*}(x, t)$ could obey compatibility conditions (of sufficiently high order) on the subsets of nonsmoothness from their domains of definition, and also the estimates (2.9) could hold for the improved function $\varphi^{*}(x, t)$.

The components from representation (2.11) satisfy the estimates

$$
\begin{aligned}
\left|\frac{\partial^{k+k_{0}}}{\partial x_{1}^{k_{1}} \partial x_{2}^{k_{2}} \partial t^{k_{0}}} U^{*}(x, t)\right| & \leqslant M\left[\sigma_{1}^{-1}+\sigma_{2}^{-1}\right]^{k+k_{0}} \\
\left|V_{j}^{*}(x, t)\right| & \leqslant M \exp \left(-m \varepsilon^{-1} r\left(x, \Gamma_{j}\right)\right) \\
\frac{\partial^{\bar{k}+k_{0}}}{\partial x_{1}^{\bar{k}_{1}} \partial x_{2}^{\bar{k}_{2}} \partial t^{k_{0}}} V_{j}^{*}(x, t) \mid & \leqslant M \varepsilon^{-\bar{k}_{1}}\left(\sigma_{1}^{-1}+\sigma_{2}^{-1}\right)^{k_{0}+2 \bar{k}_{2}+\bar{k}_{1} / 2} \\
\left|V_{j i}^{*}(x, t)\right| & \leqslant M \min _{s=j, r}\left[\exp \left(-m \varepsilon^{-1} r\left(x, \Gamma_{s}\right)\right)\right] \\
\left|\frac{\partial^{k+k_{0}}}{\partial x_{1}^{k_{1}} \partial x_{2}^{k_{2}} \partial t^{k_{0}}} V_{j i}^{*}(x, t)\right| & \leqslant M \varepsilon^{2 k_{0}}\left[\varepsilon^{-1}\left(\sigma_{1}^{-1}+\sigma_{2}^{-1}\right)^{1 / 2}+\left(\sigma_{1}^{-1}+\sigma_{2}^{-1}+r^{-1}\right)\right]^{k+2 k_{0}} \\
\left|V_{j i}^{*}(x, t)-V_{j i}^{*}\left(x^{*}, t\right)\right| & \leqslant M\left[\varepsilon^{-1} \sigma_{2}^{-1 / 2}+\sigma_{1}^{-1}+\sigma_{2}^{-1}\right] r
\end{aligned}
$$

moreover,

$$
\begin{array}{rlrl}
\left|\frac{\partial}{\partial t} U^{*}(x, t)\right| & \leqslant M \sigma_{2}^{-1}, \\
\left|\frac{\partial^{\bar{k}+k_{0}}}{\partial x_{1}^{\bar{k}_{1}} \partial x_{2}^{\bar{k}_{2}} \partial t^{k_{0}}} V_{j}^{*}(x, t)\right| & \leqslant M \sigma_{2}^{-\left(k_{0}+\bar{k}_{1} / 2\right)} \varepsilon^{-\bar{k}_{1}} & \\
\left|\frac{\partial^{k+k_{0}}}{\partial x_{1}^{k_{1}} \partial x_{2}^{k_{2}} \partial t^{k_{0}}} V_{j i}^{*}(x, t)\right| & \leqslant M \varepsilon^{2 k_{0}}\left[\varepsilon^{-1} \sigma_{2}^{-1 / 2}+\left(\sigma_{1}^{-1}+\sigma_{2}^{-1}+r^{-1}\right)\right]^{k+2 k_{0}} & \text { for } k_{0} \leqslant 1, \\
(x, t) \in \bar{G}, \quad j=1, \ldots, 4, &
\end{array}
$$


where $\bar{k}_{s}=\bar{k}_{s}\left(k_{1}, k_{2}, j\right), \bar{k}_{1}=k_{1}$ for $j=1,3, \bar{k}_{1}=k_{2}$ for $j=2,4, \bar{k}_{1}+\bar{k}_{2}=k ; \bar{k}=\bar{k}\left(\bar{k}_{1}, \bar{k}_{2}, j\right)$, $\bar{k}=\bar{k}_{1}$ for $j=1,3, \bar{k}=\bar{k}_{2}$ for $j=2,4 ; r\left(x, \Gamma_{j}\right)$ is the distance from the point $x$ to the set $\Gamma_{j}, x^{*}=x^{*}(x)$ is a point on $\Gamma$ nearest to the point $x$ (if more of such points exist, then $x^{*}$ is one of them), $m$ is an arbirtary number. The estimates (2.15) is derived in a similar way as the above estimate $(2.6)$ (see also $[3,8,13]$ ).

2.2.2. To obtain appropriate bounds for the solution of problem (1.1), (2.1), we decompose $u(x, t)$ into the sum of the three functions

$$
u(x, t)=U^{*}(x, t)+V^{*}(x, t)+v(x, t)=u^{*}(x, t)+\left(v(x, t)-v^{*}(x, t)\right), \quad(x, t) \in \bar{G},
$$

where $u^{*}(x, t)=u_{(2.10)}^{*}(x, t), U^{*}(x, t)$ and $V^{*}(x, t)$ are the components from (2.11). Let us estimate the components $v_{(2.16)}(x, t)$ and $v_{(2.11)}^{*}(x, t)$.

The functions $v^{*}(x, t)$ and $v(x, t)$ satisfy the equations

$$
\begin{aligned}
L_{(2.10)} v^{*}(x, t) & =-L_{(2.10)}\left(U^{*}(x, t)+V^{*}(x, t)\right)+f^{*}(x, t), & & (x, t) \in G, \\
v^{*}(x, t) & =0, & & (x, t) \in S ; \\
L_{(1.1)} v(x, t) & =-L_{(1.1)}\left(U^{*}(x, t)+V^{*}(x, t)\right)+f(x, t), & & (x, t) \in G, \\
v(x, t) & =\varphi(x, t)-\varphi^{*}(x, t), & & (x, t) \in S .
\end{aligned}
$$

Taking estimates (2.15) into account, we find

$$
\begin{aligned}
&\left|L_{(2.10)} U^{*}(x, t)-f^{*}(x, t)\right| \\
&\left|L_{(1.1)} U^{*}(x, t)-f(x, t)\right| \leqslant M\left[\sigma_{1}^{\alpha_{1}}+\sigma_{2}^{\alpha_{2}}+\varepsilon^{2}\left(\sigma_{1}^{-1}+\sigma_{2}^{-1}\right)^{2}\right], \\
&\left|L_{(2.10)} V_{j}^{*}(x, t)\right|,\left|L_{(1.1)} V_{j}^{*}(x, t)\right| \leqslant M\left[\sigma_{2}^{-1} \sigma_{1}^{\alpha_{1}}+\varepsilon^{\alpha_{1}}+\varepsilon^{2}\left(\sigma_{1}^{-1}+\sigma_{2}^{-1}\right)^{4}\right], \\
&\left|L_{(2.10)} V_{j i}^{*}(x, t)\right|, \quad\left|L_{(1.1)} V_{j i}^{*}(x, t)\right| \leqslant M\left[\sigma_{1}^{\alpha_{1}}+r^{\alpha_{1}}\right]\left[\sigma_{2}^{-1}+\varepsilon^{2}\left(\sigma_{1}^{-1}+\sigma_{2}^{-1}+r^{-1}\right)^{2}\right], \\
&\left|v^{*}(x, t)\right|, \quad\left|v(x, t)-v\left(x^{*}, t\right)\right| \leqslant M\left\{\sigma_{2}^{\alpha_{2}}+\varepsilon^{-1}\left[\sigma_{2}^{-1 / 2}+\varepsilon\left(\sigma_{1}^{-1}+\sigma_{2}^{-1}\right)\right] r\right\}, \\
&(x, t) \in \bar{G}, \quad x^{*}=x_{(2.15)}^{*}(x) .
\end{aligned}
$$

Hence, the components $v^{*}(x, t)$ and $v(x, t)$ satisfy

$$
\begin{aligned}
\left|v^{*}(x, t)\right|,|v(x, t)| \leqslant M\{ & \sigma_{2}^{\alpha_{2}}+\sigma_{1}^{\alpha_{1}} \sigma_{2}^{-1}+\varepsilon^{\alpha_{1}}+\varepsilon^{2}\left(\sigma_{1}^{-1}+\sigma_{2}^{-1}\right)^{4} \\
& +\left[\sigma_{1}^{\alpha_{1}}+\varepsilon^{\bar{\alpha}_{1}}\right]\left[\sigma_{2}^{-1 / 2}+\varepsilon\left(\sigma_{1}^{-1}+\sigma_{2}^{-1}+\rho^{-1}\right)\right]^{2} \\
& \left.+\varepsilon^{-1}\left[\sigma_{2}^{-1 / 2}+\varepsilon\left(\sigma_{1}^{-1}+\sigma_{2}^{-1}\right)\right] \rho\right\}, \quad(x, t) \in \bar{G},
\end{aligned}
$$

where $\bar{\alpha}_{1}$ is an arbitrary parameter from the interval $\left(0, \alpha_{1}\right), \rho>0$ is any number.

The estimate (2.17) with

$$
\rho=\varepsilon \sigma_{2}^{1 / 6}\left[\sigma_{1}^{\alpha_{1}}+\varepsilon^{\bar{\alpha}_{1}}\right]^{1 / 3}, \quad \sigma_{2}=\left[\sigma_{1}^{\alpha_{1}}+\varepsilon^{\bar{\alpha}_{1}}\right]^{1 /\left(1+3 \alpha_{2}\right)}, \quad \sigma_{1}=\varepsilon^{2\left(1+3 \alpha_{2}\right) /\left(4+12 \alpha_{2}+\alpha_{1} \alpha_{2}\right)}
$$

leads to the following bounds on the remainder terms $v_{(2.11)}^{*}(x, t)$ and $v_{(2.16)}(x, t)$ :

$$
\left|v^{*}(x, t)\right|, \quad|v(x, t)| \leqslant M \varepsilon^{\nu}, \quad(x, t) \in \bar{G},
$$


where $\nu=\nu\left(\alpha_{1}, \alpha_{2}\right) \equiv 2 \alpha_{1} \alpha_{2}\left(4+12 \alpha_{2}+\alpha_{1} \alpha_{2}\right)^{-1}, \quad \nu>9^{-1} \alpha_{1} \alpha_{2}$.

Under the smoothness condition (1.2) we have

$$
\left|v^{*}(x, t)\right|, \quad|v(x, t)| \leqslant M \varepsilon^{\nu}, \quad(x, t) \in \bar{G},
$$

where $\nu=\nu(\alpha) \equiv 2^{-1} \alpha^{2}(1+2 \alpha)^{-2}, \nu \geqslant 18^{-1} \alpha^{2}$. Thus, the function $u^{*}(x, t)$ for $\alpha_{1}=\alpha_{2}=\alpha$ is the main term in the asymptotic expansion of the solution $u(x, t)$ of problem $(1.1),(1.2)$ :

$$
\left|u(x, t)-u^{*}(x, t)\right|, \quad\left|u(x, t)-\left(U^{*}(x, t)+V^{*}(x, t)\right)\right| \leqslant M \varepsilon^{\nu}, \quad(x, t) \in \bar{G},
$$

where $\nu=\nu_{(2.19)} ; u^{*}(x, t), U^{*}(x, t), V^{*}(x, t)$ are determined for $\alpha_{1}=\alpha_{2}=\alpha, \quad \alpha=\alpha_{(1.2)}$.

We summarize these results in the following theorem.

Theorem 2.2. Let the data of problem (1.1) satisfy the smoothness condition (2.1). Then the estimates (2.15), (2.17), and (2.18) hold for the components from representation (2.11) of the function $u^{*}(x, t)$, i.e., the solution of the "similar" problem (2.10), (2.8). In the case of the smoothness condition (1.2) the solution $u(x, t)$ of problem (1.1) satisfies the estimate (2.20).

\section{Finite difference schemes for problem (2.1)}

\subsection{Nonuniform error bounds}

Let us first consider a classical finite difference method for problem (1.1) in the case of conditions (2.1) and (1.2).

3.1.1. On the set $\bar{G}$ we introduce the rectangular mesh as the tensor product of onedimensional meshes:

$$
\bar{G}_{h}=\bar{D}_{h} \times \bar{\omega}_{0}, \quad \bar{D}_{h}=\bar{\omega}_{1} \times \bar{\omega}_{2},
$$

where $\bar{\omega}_{s}$ is, generally speaking, a nonuniform mesh on the segment $\left[0, d_{s}\right]$ of the $x_{s}$ axis, $s=1,2$, and $\bar{\omega}_{0}$ is a uniform mesh on $[0, T]$ with step size $h_{t}$. Define $h_{s}^{i}=x_{s}^{i+1}-x_{s}^{i}, x_{s}^{i}$, $x_{s}^{i+1} \in \bar{\omega}_{s}, h_{s}=\max _{i} h_{s}^{i}, h=\max _{s} h_{s}, s=1,2$. By $N_{s}+1, s=1,2$ and $N_{0}+1$ we denote the number of nodes in the meshes $\bar{\omega}_{s}$ and $\bar{\omega}_{0}$, respectively; let $N=\min _{s} N_{s}, s=1,2$, and $h \leqslant M N^{-1}$.

On the mesh $\bar{G}_{h}$, we approximate problem (1.1) by the implicit finite difference scheme [11]

$$
\begin{aligned}
\Lambda_{(3.2)} z(x, t) & =f(x, t), & & (x, t) \in G_{h}, \\
z(x, t) & =\varphi(x, t), & & (x, t) \in S_{h} .
\end{aligned}
$$

Here $G_{h}=G \cap \bar{G}_{h}, \quad S_{h}=S \cap \bar{G}_{h}$,

$$
\Lambda_{(3.2)} z(x, t) \equiv\left\{\varepsilon^{2} \sum_{s=1,2} a_{s}(x, t) \delta_{\overline{x s \widehat{x}}}-c(x, t)-p(x, t) \delta_{\bar{t}}\right\} z(x, t)
$$

$\delta_{\overline{x s} \widehat{x s}} z(x, t)$ and $\delta_{\bar{t}} z(x, t)$ are the second (central) and first (backward) finite differences; for example, $\delta_{\overline{x 1} \widehat{x 1}} z(x, t)=2\left(h_{1}^{i-1}+h_{1}^{i}\right)^{-1}\left(\delta_{x 1} z(x, t)-\delta_{\overline{x 1}} z(x, t)\right), x_{1}=x_{1}^{i}$.

The maximum principle [11] is valid for the difference scheme (3.2), (3.1). 
3.1.2. Let us estimate the error $|u(x, t)-z(x, t)|,(x, t) \in \bar{G}_{h}$ in the case of condition (2.1).

Assume that some function $w(x, t),(x, t) \in \bar{G}$ satisfies the condition $w \in C^{2,1}(G) \cap C(\bar{G})$. Let $z_{w}(x, t),(x, t) \in \bar{G}_{h}$ denote the solution of the problem

$$
\begin{aligned}
\Lambda_{(3.2)} z(x, t) & =L_{(1.1)} w(x, t), & & (x, t) \in G_{h}, \\
z(x, t) & =w(x, t), & & (x, t) \in S_{h} .
\end{aligned}
$$

According to this notation and the decomposition (2.4), we represent the solution of scheme (3.2), (3.1) in the form

$$
z(x, t)=z_{u^{1}}(x, t)+z_{v^{1}}(x, t), \quad(x, t) \in \bar{G}_{h},
$$

where $u^{1}(x, t)=u_{(2.3)}^{1}(x, t), v^{1}(x, t)=v_{(2.4)}^{1}(x, t)$. It follows from the maximum principle that

$$
\left|z(x, t)-z_{u^{1}}(x, t)\right|, \quad\left|z_{v^{1}}(x, t)\right| \leqslant M \sigma_{0}^{\alpha_{2}}, \quad|z(x, t)| \leqslant M, \quad(x, t) \in \bar{G}_{h} .
$$

Taking into account the a-priori estimates of the function $u^{1}(x, t)$ (see $(2.6),(2.7)$ ) we obtain the following inequalities for the function $z_{u^{1}}(x, t)$ :

$$
\begin{array}{rlrl}
\left|\Lambda_{(3.2)}\left(u^{1}(x, t)-z_{u^{1}}(x, t)\right)\right| & =\left|\left(\Lambda_{(3.2)}-L_{(1.1)}\right) u^{1}(x, t)\right| \\
& \leqslant M\left[\varepsilon r^{-1}+t^{-1 / 2}\right]^{2+\alpha_{1}}\left[\varepsilon^{-1} N^{-1}+N_{0}^{-1 / 2}\right]^{\alpha_{1}}, & & (x, t) \in G_{h}, \\
\left|u^{1}(x, t)-z_{u^{1}}(x, t)\right| & \leqslant M\left(\varepsilon+\sigma_{0}^{1 / 2}\right) \sigma_{0}^{-1} \min \left[\varepsilon^{-1} r,\left(\varepsilon+\sigma_{0}^{1 / 2}\right) \sigma_{0}^{-1} t\right], \quad(x, t) \in \bar{G}_{h},
\end{array}
$$

which imply

$$
\begin{aligned}
\left|u^{1}(x, t)-z_{u^{1}}(x, t)\right| \leqslant M\{ & {\left[\varepsilon \rho^{-1}+\tau^{-1 / 2}\right]^{2+\alpha_{1}}\left[\varepsilon^{-1} N^{-1}+N_{0}^{-1 / 2}\right]^{\alpha_{1}} } \\
& \left.+\left(\varepsilon+\sigma_{0}^{1 / 2}\right) \sigma_{0}^{-1} \min \left[\varepsilon^{-1} \rho,\left(\varepsilon+\sigma_{0}^{1 / 2}\right) \sigma_{0}^{-1} \tau\right]\right\}, \quad(x, t) \in \bar{G}_{h},
\end{aligned}
$$

where $\rho, \tau>0$ are arbitrary numbers. By (2.5) and (3.3) we find

$$
\begin{aligned}
|u(x, t)-z(x, t)| \leqslant M\{ & \sigma_{0}^{\alpha_{2}}+\left[\varepsilon \rho^{-1}+\tau^{-1 / 2}\right]^{2+\alpha_{1}}\left[\varepsilon^{-1} N^{-1}+N_{0}^{-1 / 2}\right]^{\alpha_{1}} \\
& \left.+\left(\varepsilon+\sigma_{0}^{1 / 2}\right) \sigma_{0}^{-1} \min \left[\varepsilon^{-1} \rho,\left(\varepsilon+\sigma_{0}^{1 / 2}\right) \sigma_{0}^{-1} \tau\right]\right\}, \quad(x, t) \in \bar{G}_{h} .
\end{aligned}
$$

If the values $\rho, \tau$ and $\sigma_{0}$ are defined by

$$
\begin{aligned}
\rho & =\varepsilon\left[\left(\varepsilon+\sigma_{0}^{1 / 2}\right) \sigma_{0}^{-1}\right]^{-1 /\left(3+\alpha_{1}\right)}\left[\varepsilon^{-1} N^{-1}+N_{0}^{-1 / 2}\right]^{\alpha_{1} /\left(3+\alpha_{1}\right)}, \\
\tau & =\left[\left(\varepsilon+\sigma_{0}^{1 / 2}\right) \sigma_{0}^{-1}\right]^{-\left(4+\alpha_{1}\right) /\left(3+\alpha_{1}\right)}\left[\varepsilon^{-1} N^{-1}+N_{0}^{-1 / 2}\right]^{\alpha_{1} /\left(3+\alpha_{1}\right)}, \\
\sigma_{0} & = \begin{cases}\varepsilon^{\left(2+\alpha_{1}\right) /\left(2+\alpha_{1}+3 \alpha_{2}+\alpha_{1} \alpha_{2}\right)}\left[\varepsilon^{-1} N^{-1}+N_{0}^{-1 / 2}\right]^{\alpha_{1} /\left(2+\alpha_{1}+3 \alpha_{2}+\alpha_{1} \alpha_{2}\right)} & \text { for } \varepsilon^{2} \geqslant \sigma_{0}, \\
{\left[\varepsilon^{-1} N^{-1}+N_{0}^{-1 / 2}\right]^{2 \alpha_{1} /\left(2+\alpha_{1}+6 \alpha_{2}+2 \alpha_{1} \alpha_{2}\right)}} & \text { for } \varepsilon^{2}<\sigma_{0},\end{cases}
\end{aligned}
$$


it follows from (3.4) that the difference scheme (3.2), (3.1) converges for fixed values of the parameter $\varepsilon$ :

$$
\begin{aligned}
|u(x, t)-z(x, t)| \leqslant M\{ & \varepsilon^{\gamma_{1} \alpha_{2}\left(2+\alpha_{1}\right)}\left[\varepsilon^{-1} N^{-1}+N_{0}^{-1 / 2}\right]^{\gamma_{1} \alpha_{1} \alpha_{2}} \\
\left.+\left[\varepsilon^{-1} N^{-1}+N_{0}^{-1 / 2}\right]^{2 \gamma_{2} \alpha_{1} \alpha_{2}}\right\}, \quad & (x, t) \in \bar{G}_{h} \\
|u(x, t)-z(x, t)| \leqslant M\left[\varepsilon^{-1} N^{-1}+N_{0}^{-1 / 2}\right]^{\alpha_{1} \alpha_{2} / 7}, & (x, t) \in \bar{G}_{h},
\end{aligned}
$$

where $\gamma_{1}=\gamma_{1}\left(\alpha_{1}, \alpha_{2}\right)=2+\alpha_{1}+3 \alpha_{2}+\alpha_{1} \alpha_{2}, \gamma_{2}=\gamma_{2}\left(\alpha_{1}, \alpha_{2}\right)=2+\alpha_{1}+6 \alpha_{2}+2 \alpha_{1} \alpha_{2}$. Recall that these estimates hold under the smoothness condition (2.1).

In the case of condition (1.2) we have

$$
|u(x, t)-z(x, t)| \leqslant M\left[\varepsilon^{-1} N^{-1}+N_{0}^{-1 / 2}\right]^{\nu}, \quad(x, t) \in \bar{G}_{h}
$$

where $\nu=\nu(\alpha)=7^{-1} \alpha^{2}, \quad \alpha=\alpha_{(1.2)}$.

Thus, under the smoothness condition (1.2) the difference scheme (3.2), (3.1) also converges for fixed values of the parameter $\varepsilon$, with an error bound given by

$$
|u(x, t)-z(x, t)| \leqslant Q(\varepsilon)\left[N^{-1}+N_{0}^{-1 / 2}\right]^{\nu}, \quad(x, t) \in \bar{G}_{h}, \quad \nu=\nu_{(3.7)},
$$

where $Q(\varepsilon)=M \varepsilon^{-\nu}$.

It follows from estimates (3.6) and (3.7) that the scheme converges under the condition

$$
\varepsilon \in\left[\varepsilon_{0}(N), 1\right], \quad \varepsilon_{0}(N) \rightarrow 0 \text { for } \quad N \rightarrow \infty
$$

if the relation

$$
\varepsilon_{0}^{-1}(N)=o(N) \text { for } \quad N \rightarrow \infty .
$$

is true. We can now state the following convergence result for the classical difference scheme, which is clearly not $\varepsilon$-uniform.

Theorem 3.1. Suppose that conditions (1.2) or (2.1) hold for the data of the boundaryvalue problem (1.1). Then the solution $z(x, t)$ of the difference scheme (3.2), (3.1) converges to the solution $u(x, t)$ of problem (1.1) for fixed values of the parameter $\varepsilon$ (more precisely, for $\varepsilon$ satisfying condition (3.9)), and the error estimates (3.4)-(3.8) are valid.

Remark 2. In estimates (3.7) and (3.8) the order of convergence is too small, comparable with $\mathcal{O}\left(\alpha^{2}\right)$, if the data of problem (1.1) have small smoothness. However, in the case of condition (2.1), when either $\alpha_{1}$ or $\alpha_{2}$ is finite, we have essentially better convergence (see (3.6)) of the order $\mathcal{O}\left(\alpha_{1}\right)$ for $\alpha_{2} \geqslant m$, or $\mathcal{O}\left(\alpha_{2}\right)$ for $\alpha_{1} \geqslant m$.

\subsection{Parameter-uniform error bounds}

To solve problem (1.1), (2.1) for arbitrarily small values of the parameter $\varepsilon$, a special mesh, which is condensed in a neighborhood of the boundary layer, is required. 
3.2.1. On the set $\bar{G}$ we introduce the piecewise uniform rectangular grid

$$
\bar{G}_{h}=\bar{D}_{h}^{*} \times \bar{\omega}_{0}, \quad \bar{D}_{h}^{*}=\bar{\omega}_{1}^{*} \times \bar{\omega}_{2}^{*}
$$

where $\bar{\omega}_{0}=\bar{\omega}_{0(3.1)}$ as before, $\bar{\omega}_{s}^{*}=\bar{\omega}_{s}^{*}\left(\sigma_{s}\right), s=1,2$ is a piecewise uniform mesh on $\left[0, d_{s}\right], \sigma_{s}$ is a mesh parameter depending on $\varepsilon$ and $N_{s}$. To construct the mesh $\bar{\omega}_{s}^{*}$, we divide the interval $\left[0, d_{s}\right]$ of the $x_{s}$ axis into three subintervals $\left[0, \sigma_{s}\right],\left[\sigma_{s}, d_{s}-\sigma_{s}\right]$, and $\left[d_{s}-\sigma_{s}, d_{s}\right]$. In each part we place a uniform mesh, with constant step sizes $h_{s}^{(1)}=4 \sigma_{s} N_{s}^{-1}$ on the subintervals $\left[0, \sigma_{s}\right],\left[d_{s}-\sigma_{s}, d_{s}\right]$, and $h_{s}^{(2)}=2\left(d_{s}-2 \sigma_{s}\right) N_{s}^{-1}$ on $\left[\sigma_{s}, d_{s}-\sigma_{s}\right]$. Following the principles set up in $[8,13]$ and $[3]$, the transition point $\sigma_{s}$ is fitted to the left-hand and right-hand boundary layers along the $x_{s}$ axis by defining

$$
\sigma_{s}=\min \left[4^{-1} d_{s}, \varepsilon m^{-1} \ln N_{s}\right], \quad s=1,2,
$$

where $m=m_{(2.15)}$. In this way the mesh $\bar{G}_{h}$ has been constructed.

Note that the estimates (3.4)-(3.8) hold for the difference scheme (3.2), (3.10).

3.2.2. We now study the behavior of the numerical solutions by scheme (3.2), (3.10) for small values of the parameter. For this we represent the solution $z(x, t)$ of this scheme in the form of a sum

$$
\begin{aligned}
z(x, t) & =z_{U^{*}}(x, t)+\sum_{j=1}^{4} z_{V_{j}^{*}}(x, t)+\sum_{j=1}^{4} z_{V_{j i}^{*}}(x, t)+z_{v}(x, t) \\
& =z_{u^{*}}(x, t)+z_{v}(x, t), \quad(x, t) \in \bar{G}_{h},
\end{aligned}
$$

where $U^{*}(x, t), V_{j}^{*}(x, t)$, and $V_{j i}^{*}(x, t)$ are the components from representation (2.11) of the function $u^{*}(x, t)$, which is the "smooth" approximation to the solution $u(x, t)$ of problem (1.1), $(2.1) ; v(x, t)=v_{(2.16)}(x, t)$ is the remainder term in representation (2.16) of the function $u(x, t)$.

Taking into account the relations

$$
\begin{aligned}
\left|\Lambda_{(3.2)} z_{v}(x, t)\right| & =\left|L_{(1.1)} v(x, t)\right|=\left|L_{(1.1)}\left(U^{*}(x, t)+V^{*}(x, t)\right)-f(x, t)\right|, & & (x, t) \in G_{h}, \\
\left|z_{v}(x, t)\right| & =|v(x, t)|=\left|\varphi(x, t)-\varphi_{(2.8)}^{*}(x, t)\right|, & & (x, t) \in S_{h},
\end{aligned}
$$

and also estimates $(2.9),(2.15)$, we obtain the following estimate of the function $z_{v}(x, t)$, similar to estimate $(2.17)$ for $v(x, t)$ :

$$
\begin{aligned}
\left|z_{v}(x, t)\right| \leqslant M\{ & \sigma_{2}^{\alpha_{2}}+\sigma_{1}^{\alpha_{1}} \sigma_{2}^{-1}+\varepsilon^{\alpha_{1}}+\varepsilon^{2}\left(\sigma_{1}^{-1}+\sigma_{2}^{-1}\right)^{4} \\
& +\left[\sigma_{1}^{\alpha_{1}}+\varepsilon^{\bar{\alpha}_{1}}\right]\left[\sigma_{2}^{-1 / 2}+\varepsilon\left(\sigma_{1}^{-1}+\sigma_{2}^{-1}+\rho^{-1}\right)\right]^{2} \\
& \left.+\varepsilon^{-1}\left[\sigma_{2}^{-1 / 2}+\varepsilon\left(\sigma_{1}^{-1}+\sigma_{2}^{-1}\right)\right] \rho\right\}, \quad(x, t) \in \bar{G}_{h}
\end{aligned}
$$

where $\bar{\alpha}_{1}=\bar{\alpha}_{1(2.17)}, \quad \alpha_{i}=\alpha_{i(2.1)}, \quad i=1,2$. 
Then we find

$$
\begin{aligned}
\left|z_{u^{*}}(x, t)-u^{*}(x, t)\right| \leqslant M\{ & \varepsilon^{2}\left(\sigma_{1}^{-1}+\sigma_{2}^{-1}\right)^{6} N^{-1}+\left[\varepsilon^{3} \rho^{-3}+\left(\sigma_{1}^{-1}+\sigma_{2}^{-1}\right)^{3}\right] N^{-1} \ln N \\
& +\left[\varepsilon^{4} \rho^{-4}+\left(\sigma_{1}^{-1}+\sigma_{2}^{-1}\right)^{4}\right] N_{0}^{-1} \\
& \left.+\rho \varepsilon^{-1}\left[\sigma_{2}^{-1 / 2}+\varepsilon\left(\sigma_{1}^{-1}+\sigma_{2}^{-1}\right)\right]\right\}, \quad(x, t) \in \bar{G}_{h} .
\end{aligned}
$$

This estimate and (2.17), (3.12) imply the estimate

$$
\begin{aligned}
|u(x, t)-z(x, t)| \leqslant M\{ & \sigma_{2}^{\alpha_{2}}+\sigma_{1}^{\alpha_{1}} \sigma_{2}^{-1}+\varepsilon^{\bar{\alpha}_{1}} \sigma_{2}^{-1}+\varepsilon^{2}\left(\sigma_{1}^{-1}+\sigma_{2}^{-1}\right)^{4} \\
& +\left[\sigma_{1}^{\alpha_{1}}+\varepsilon^{\bar{\alpha}_{1}}+N^{-1}+N_{0}^{-1}\right] \varepsilon^{4} \rho^{-4} \\
& +\rho \varepsilon^{-1}\left[\sigma_{2}^{-1 / 2}+\varepsilon\left(\sigma_{1}^{-1}+\sigma_{2}^{-1}\right)\right] \\
& \left.+\varepsilon^{2}\left(\sigma_{1}^{-1}+\sigma_{2}^{-1}\right)^{6} N^{-1}+\left(\sigma_{1}^{-1}+\sigma_{2}^{-1}\right)^{4} N_{0}^{-1}\right\}, \quad(x, t) \in \bar{G}_{h} .
\end{aligned}
$$

Choosing the parameters $\rho, \sigma_{1}$ and $\sigma_{2}$ as follows

$$
\begin{aligned}
\rho & =\varepsilon \sigma_{2}^{1 / 10}\left[\sigma_{1}^{\alpha_{1}}+\varepsilon^{\bar{\alpha}_{1}}+N^{-1}+N_{0}^{-1}\right]^{1 / 5}, \\
\sigma_{2} & =\sigma_{1}^{\alpha_{1} /\left(2+5 \alpha_{2}\right)} \\
\sigma_{1} & =\left[\varepsilon^{2}+N^{-1}+N_{0}^{-1}\right]^{\alpha_{1} \alpha_{2} /\left(8+20 \alpha_{2}+\alpha_{1} \alpha_{2}\right)},
\end{aligned}
$$

we obtain the error estimate

$$
|u(x, t)-z(x, t)| \leqslant M\left[\varepsilon^{2}+N^{-1}+N_{0}^{-1}\right]^{\alpha_{1} \alpha_{2} /\left[10\left(1+2 \alpha_{2}\right)\right]}, \quad(x, t) \in \bar{G}_{h} .
$$

It follows from (3.6), (3.13) that the scheme (3.2), (3.10) converges $\varepsilon$-uniformly:

$$
|u(x, t)-z(x, t)| \leqslant M\left[N^{-1}+N_{0}^{-1}\right]^{\nu}, \quad(x, t) \in \bar{G}_{h},
$$

where $\nu=\nu\left(\alpha_{1}, \alpha_{2}\right) \equiv 4^{-1} \alpha_{1} \alpha_{2}\left(4+5 \alpha_{2}\right)^{-1}, \quad \nu \geqslant 36^{-1} \alpha_{1} \alpha_{2}$. Of course, this convergence result occurs under the assumption that the smoothness condition (2.1) is valid.

If the original condition (1.2) is true, the following $\varepsilon$-uniform error estimate holds:

$$
|u(x, t)-z(x, t)| \leqslant M\left[N^{-1}+N_{0}^{-1}\right]^{\nu}, \quad(x, t) \in \bar{G}_{h}
$$

where $\nu=\nu(\alpha) \equiv 4^{-1} \alpha^{2}(4+5 \alpha)^{-1}, \nu>36^{-1} \alpha^{2}$.

We want to stress that, near the corner points, the analytic behavior of the corner boundary layer function can be more complicated if the data of the problem are not sufficiently smooth and compatible. This is a remarkable feature of the piecewice uniform fitted mesh $\bar{G}_{h(3.10)}$ that, without modification of the mesh in the corner, it leads to numerical solutions that converge $\varepsilon$-uniformly even in this corner layer. We are thus led to the following main result. 
Theorem 3.2. Let conditions (1.2) or (2.1) hold for the data of the boundary-value problem (1.1). Then the solution $z(x, t)$ of the difference scheme (3.2), (3.10) converges $\varepsilon$-uniformly to the solution $u(x, t)$ of problem (1.1). The error estimates (3.6), (3.7) and (3.13)-(3.15) are valid for the discrete solutions.

Note that the rate of $\varepsilon$-uniform convergence of the difference scheme (3.2), (3.10) depends on the parameters $\alpha_{1}$ and $\alpha_{2}$ in much the same way as has been observed in Remark 2 to Theorem 3.1.

\section{Generalizations and remarks}

4.1. It may turn out that the data of the boundary-value problem (1.1) on some part of $\bar{G}$ are smoother in comparison with the same data on the whole domain $\bar{G}$. Under certain conditions this fact implies that the solution of the boundary-value problem becomes smoother and, consequently, this results in an increased order of convergence of the numerical solution on a subdomain in that part where the data are smoother. Let us consider such a problem.

Let the smoothness condition (1.2) hold for the data of the boundary-value problem (1.1) and, in addition, let us assume that, on some subset $\bar{G}^{0}, G^{0} \subseteq G$, the data of problem (1.1) are smoother than in (1.2). Besides, the boundary function may satisfy additional compatibility conditions on the intersection of the faces $S_{j}$ which belong to $S^{0}$ ( $S^{0}$ is the parabolic boundary of the set $\left.G^{0}\right)$. Let the data of problem (1.1) be extended from $\bar{G}^{0}$ onto $\bar{G}$ with the preservation of their increased smoothness, and assume that the difference $u^{*}(x, t)-z^{*}(x, t),(x, t) \in \bar{G}_{h}$ satisfies the estimate

$$
\left|u^{*}(x, t)-z^{*}(x, t)\right| \leqslant M\left[N^{-1}+N_{0}^{-1}\right]^{\beta}, \quad(x, t) \in \bar{G}_{h},
$$

where $\beta$ is some constant from the half-interval $(0,1] ; u^{*}(x, t),(x, t) \in \bar{G}$ and $z^{*}(x, t)$, $(x, t) \in \bar{G}_{h}$ denote the solutions of the boundary-value problem with the smoother data on $\bar{G}$ and of the corresponding discrete problem on $\bar{G}_{h(3.10)}$.

We try to find the subset $\bar{G}^{*}$ of $\bar{G}^{0}$, on which the following estimate is fulfilled for sufficiently small values of the parameter $\varepsilon$ :

$$
|u(x, t)-z(x, t)| \leqslant M\left[N^{-1}+N_{0}^{-1}\right]^{\beta}, \quad(x, t) \in \bar{G}_{h}^{*},
$$

where $\bar{G}_{h}^{*}=\bar{G}^{*} \cap \bar{G}_{h(3.10)}$. Note that the solution $z(x, t)$ of scheme (3.2), (3.10) satisfies the estimate (3.15); assume $\beta>\nu_{(3.15)}$.

We construct the set $\bar{G}^{*}$ as follows. For a point $\left(x^{0}, t^{0}\right) \in \bar{G}$ we introduce its $\left(\delta_{1}, \delta_{2}\right)$ vicinity, that is, the set $H\left(x^{0}, t^{0} ; \delta_{1}, \delta_{2}\right)=\left\{(x, t): x_{s}^{0}-\delta_{s}<x_{s}<x_{s}^{0}+\delta_{s}, s=1,2, t=t^{0}\right\}$. Let the set $\bar{G}^{1}$ be formed by connected line segments (parallel to the axis $t$ ) entirely lying in $\bar{G}^{0}$, one endpoint of which belongs to the lower base $S_{0}$. By $\bar{G}^{1}\left(\delta_{1}, \delta_{2}\right)$ we denote the set of the points from $\bar{G}^{1}$ that are not contained in an $\left(\delta_{1}, \delta_{2}\right)$-vicinity of the set $S^{1} \backslash\left\{S^{0} \cap S\right\}$, where $S^{1}$ is the parabolic boundary of the set $G^{1}$. The set $\bar{G}^{1}\left(\delta_{1}, \delta_{2}\right)$ adjoins the set $S_{0}$, however, it may not adjoin the set $S^{L}$. Finally, the set $\bar{G}^{*}$ we want to locate is the set $\bar{G}^{1}\left(\delta_{1}, \delta_{2}\right)$ :

$$
\bar{G}^{*}=\bar{G}^{1}\left(\delta_{1}, \delta_{2}\right)
$$

for the values of the parameters $\delta_{s}=\delta_{s}\left(\varepsilon, N, N_{0}\right)$ chosen below. 
Using the majorant functions, we ascertain that the estimate (4.2) is fulfilled under the condition

$$
\delta_{1}=\delta_{2}=\delta \equiv \ln ^{-1} q N^{-1} \ln N, \quad q=1+\varepsilon^{-1} N^{-1}+\varepsilon^{-2} N^{-2} .
$$

Note that the quantity $\delta_{(4.4)}$ tends to zero provided that $\varepsilon \leqslant \varepsilon_{0}(N)$, if $\varepsilon_{0}(N) \ln N \rightarrow 0$ as $N \rightarrow \infty$. Under the condition

$$
\varepsilon=o\left(N^{-1} \ln ^{-1} N\right), \quad N \rightarrow \infty
$$

the value of $\delta_{s}$ is defined by

$$
\delta_{s}=h_{s}^{(2)}, \quad s=1,2, \quad h_{s}^{(2)}=h_{s(3.10)}^{(2)},
$$

that is, under condition (4.5a) the scheme (3.2), (3.10) converges $\varepsilon$-uniformly with the rate $\mathcal{O}\left(N^{-\beta}+N_{0}^{-\beta}\right)$ at all the nodes from $\bar{G}_{h}^{1}=\bar{G}^{1} \cap \bar{G}_{h}$ that do not fall into the $\left(h_{1}^{(2)}, h_{2}^{(2)}\right)$ vicinity of the set $S^{1} \backslash S$.

4.2. In the case where the data of problem (1.1) extended from $\bar{G}^{0}$ onto the whole set $\bar{G}$ are sufficienty smooth (see, e.g., Section 1 and also [13]), the estimate similar to (4.1) holds for the solutions of scheme (3.2), (3.10):

$$
|u(x, t)-z(x, t)| \leqslant M\left[N^{-1} \ln N+N_{0}^{-1}\right]^{\nu}, \quad(x, t) \in \bar{G}_{h} .
$$

Here $\nu=1$, if appropriate compatibility conditions are imposed at the intersection of the faces $\bar{S}_{j}$, and $\nu=5^{-1}$ otherwise. The compatibility conditions hold, for example, if we take $\varphi(x, t) \equiv 0,(x, t) \in S$, when

$$
\frac{\partial^{k+k_{0}}}{\partial x_{1}^{k_{1}} \partial x_{2}^{k_{2}} \partial t^{k_{0}}} f(x, t)=0, \quad(x, t) \in S_{E}, k+2 k_{0} \leqslant 6 .
$$

To get the estimate (4.1), we can use the estimate (3.15), where the magnitude of $\alpha$ corresponds to the smoother data of problem (1.1) on the subset $\bar{G}^{0}$ of $\bar{G}$.

4.3. Let the data of problem (1.1) be such that the solution of scheme (3.2), (3.10), generally speaking, does not converge on $\bar{G}_{h}$, but the solutions of problems (1.1) and (3.2), (3.10) exist and are $\varepsilon$-uniformly bounded (for example, when the data have discontinuities). Assume that the data of the problem are sufficiently smooth on $\bar{G}^{0} \subset \bar{G}$ and admit the validity of estimate (4.1) (under appropriate smooth extension of the data beyond $\bar{G}^{0}$ ). In this case, under condition (4.4), the error estimate (4.2) holds on the set $\bar{G}_{(4.3)}^{*}$, where $\beta$ is defined by the smoothness of the data on $\bar{G}^{0}$ and also by imposing compatibility conditions on nonsmooth parts of the boundary $S$.

4.4. If the data of problem (1.1) satisfy the condition

$$
a_{s}, p, c, f \in C^{\alpha}(\bar{G}), \quad \varphi \in C(S), \quad s=1,2, \quad \alpha>0,
$$

then the technique given in this paper allows us to prove the $\varepsilon$-uniform convergence of scheme (3.2), (3.10):

$$
|u(x, t)-z(x, t)| \leqslant M \lambda\left(N, N_{0}\right), \quad(x, t) \in \bar{G}_{h},
$$


where the quantity $\lambda\left(N, N_{0}\right) \rightarrow 0$-uniformly as $N, N_{0} \rightarrow \infty$. Note that the solution of problem (1.1) under the smoothness condition (4.6) belongs only to the class $C^{2,1}(G) \cap C(\bar{G})$; the quantity $\lambda\left(N, N_{0}\right)$ depends on modules of the uniform continuity of the function $\varphi(x, t)$.

4.5. The results obtained for singularly perturbed parabolic reaction-diffusion equations can be generalized easily onto stationary problems for singularly perturbed elliptic equations. For such problems the a-priori estimates (bounds on the derivatives with respect to the space variables) remain valid, when replacing $m_{(2.15)}$ by the constant $m_{(2.15)}^{\star}$, which takes arbitrary values from the interval $\left(0, m_{0}\right)$, where $m_{0}=\left[\min _{\bar{D}, s}\left(a_{s}^{-1}(x) c(x)\right)\right]^{1 / 2}$, and $c(x) \geqslant c_{0}>0$, $x \in \bar{D}$. We emphasize that this constant $m_{(2.15)}^{\star}$ defines the piecewise uniform mesh $\bar{D}_{h(3.10)}^{*}$, on which we consider a monotone classical approximation of the stationary boundary-value problem.

The author is indebted to E. O'Riordan and P.N. Vabishchevich for helpful discussions of numerical methods for problems with nonsmooth solutions.

\section{References}

[1] N. S. Bakhvalov, On the optimization of methods for solving boundary-value problems with a boundary layer, Zh. Vychisl. Mat. Mat. Fiz., 9 (1969), No. 4, pp. 841-859, in Russian.

[2] E. P. Doolan, J. J. H. Miller, and W. H. A. Schilders, Uniform Numerical Methods for Problems with Initial and Boundary Layers, Boole Press, Dublin, 1980.

[3] P. A. Farrell, A. F. Hegarty, J. J. H. Miller, E. O'Riordan, and G. I. Shishkin, Robust Computational Techniques for Boundary Layers, Chapman \& Hall/CRC, Boca Raton, FL, 2000.

[4] A. M. Il'in, Differencing scheme for a differential equation with a small parameter at the highest derivative, Math. Notes, 6 (1969), No. 2, pp. 596-602.

[5] A. M. Il'in, Matching of Asymptotic Expansions of Solutions of Boundary Value Problems, Nauka, Moscow, 1989, in Russian; Translations of Mathematical Monographs, 102, American Mathematical Society, Providence, RI, 1992.

[6] O. A. Ladyzhenskaya, V. A. Solonnikiv, and N. N. Ural'tseva, Linear and Quasilinear Equations of Parabolic Type, Nauka, Moscow, 1967, in Russian; Translations of Mathematical Monographs, 23, American Mathematical Society, Providence, RI, 1968.

[7] G. I. Marchuk, Methods of Numerical Mathematics, 2nd edn., Springer-Verlag, New York-Berlin, 1982.

[8] J. J. H. Miller, E. O'Riordan, and G. I. Shishkin, Fitted Numerical Methods for Singular Perturbation Problems. Error Estimates in the Maximum Norm for Linear Problems in One and Two Dimensions, World Scientific, Singapore, 1996.

[9] K. W. Morton, Numerical Solution of Convection-Diffusion Problems, Chapman \& Hall, London, 1996.

[10] H. G. Roos, M. Stynes, and L. Tobiska, Numerical Methods for Singularly Perturbed Differential Equations. Convection-Diffusion and Flow Problems, Springer-Verlag, Berlin, 1996.

[11] A. A. Samarskii, Theory of Difference Schemes, 3rd edn., Nauka, Moscow, 1989, in Russian; English transl.: The Theory of Difference Schemes Marcel Dekker, Inc., New York, 2001. 
[12] G. I. Shishkin, Approximation of solutions of singularly perturbed boundary-value problems with a corner boundary layer, Zh. Vychisl. Mat. Mat. Fiz., 27 (1987), No. 9, pp. 1360-1374, in Russian.

[13] G. I. Shishkin, Grid Approximations of Singularly Perturbed Elliptic and Parabolic Equations, Ural Branch of Russian Acad. Sci., Ekaterinburg, 1992, in Russian.

[14] G. I. Shishkin, Approximation of singularly perturbed convection-diffusion equations with low smoothness of the derivatives involved in the equation, in: Analytical and Numerical Methods for ConvectionDominated and Singularly Perturbed Problems (L. G. Vulkov, J. J. H. Miller, and G. I. Shishkin, eds.), Nova Science Publishers, Huntington, NY, 2000, pp. 111-121.

[15] V. A. Steklov, On asymptotic expressions for certain functions defined by linear second-order differential equations and their application to a problem of decomposition of one arbitrary function into a series of these functions, Kharkov, 1956, pp. 1-138, in Russian.

[16] V. A. Steklov, Fundamental Problems in Mathematical Physics, 2nd edn., (V. S. Vladimirov, ed.), Nauka, Moscow, 1983, in Russian.

Received 14 Feb. 2001

Revised 30 Aug. 2001 